\title{
Luchas campesinas y resistencia frente a los conflictos ambientales en la Zona de Reserva Campesina de Cabrera, Cundinamarca: una alternativa territorial para la paz $^{2}$
}

\author{
Farmer struggles and strength on facing environmental conflicts in the \\ Rural Reserve Area of Cabrera, Cundinamarca: a territorial alternative to peace.
}

\begin{abstract}
Resumen
Este artículo analiza los conflictos ambientales presentes en la Zona de Reserva Campesina (ZRC) de Cabrera, Cundinamarca, como parte de la construcción social del territorio. En la ZRC existen actualmente ciertas dificultades en el ámbito socioambiental que han puesto en alerta a la comunidad de Cabrera: hay una clara tensión por la construcción de un proyecto hidroeléctrico que se planea en buena parte del territorio de la ZRC. A causa de dicho megaproyecto, la comunidad cabreruna ha empezado a replantear la visión que tiene del entorno que habita, de sus prácticas, y ha creado también estrategias de resistencia. Ello convierte en actores fundamentales a las organizaciones campesinas, que a su vez promueven la perspectiva de considerar la ZRC de Cabrera como una forma alternativa de construcción territorial para la paz.
\end{abstract}

Palabras clave: Zona de Reserva Campesina, conflictos ambientales, territorio y paz, organización rural local.

\section{Abstract}

This paper analyzes environmental conflicts arisen in the Rural Reserve Area (RRA) of Cabrera, Cundinamarca, as a way of building social territory. One of the aims of Rural Reserve Area is the care and protection of the strategic ecosystem of the municipality. However, it has been experienced currently some socioenvironmental issues that have set Cabrera community on alert: there has been a clear tension due to a bydroelectric megaproject that is underway and threatens farmer practices, forcing them to be more awareness about their environment and developing some claim strategies. So, farmer organizations have becoming essential agents on promoting Rural Reserve Area as way of building a territorial alternative to peace.

Keywords: Rural Reserve Area, environmental conflict, territory and peace, rural local organization

Recibido: 3 julio de 2015, evaluado: 15 de julio de 2015, aprobado: 24 de julio de 2015

1 Geógrafa por la Universidad Nacional de Colombia. Se dedicada especialmente al estudio de temas relacionados con el ordenamiento territorial y los conflictos ambientales. Correo electrónico: bcsilvav@unal.edu.co

2 El artículo se deriva del trabajo de grado Territorio y conflictos ambientales en la Zona de Reserva de Cabrera, Cundinamarca, (2000-2014), del programa de Geografía de la Universidad Nacional de Colombia. Este trabajo se enmarcó, a su vez, en el proceso de investigación y acompañamiento a la comunidad campesina de Cabrera, auspiciado por el grupo de investigación Ciudadanía, Paz y Desarrollo de la Corporación Universitaria Minuto de Dios y el Departamento de Lenguajes y Estudios Socioculturales de la Universidad de los Andes, durante el periodo 2012-2014. 


\section{Introducción}

Las Zonas de Reserva Campesina (en adelante, ZRC) se constituyen en el país a través de la Ley 160 de 1994, "por medio de la cual se crea el Sistema Nacional de Reforma Agraria y Desarrollo Rural Campesino, se establece un subsidio para la adquisición de tierras, se reforma el Instituto Colombiano de Reforma Agraria y se dictan otras disposiciones". De esta manera, se crea la ZRC como una figura que reconoce legalmente regiones con particularidades agroecológicas y socioeconómicas, como objeto de ordenamiento ambiental y que permite la garantía de los "derechos sociales, económicos y culturales de los campesinos, su participación en las instancias de planificación y decisión regionales y las características de las modalidades de producción" (artículo 80). Sin embargo, ese reconocimiento ha estado obstaculizado debido a que las distintas regiones del país están afectadas por el conflicto armado, la repartición inadecuada de tierras y la creciente vulneración de los recursos ambientales.

A partir de dichas condiciones, en el 2000 el municipio Cabrera, ubicado en el departamento de Cundinamarca, se constituye formalmente como ZRC. Así, se convierte en uno de los ejemplos de intervención política por parte del campesinado colombiano y contribuye a la superación del desconocimiento histórico que en el país se ha tenido de los grupos étnicos y campesinos en relación con la construcción de sus territorios.

La provincia del Sumapaz, de la cual forma parte el municipio de Cabrera, alberga el páramo del Sumapaz —el más grande del mundo-, uno de los sitios de alta montaña más rico en especies de flora y fauna colombiana, con un sinnúmero de organismos que se preservan debido a su importancia ambiental y a la diversidad biológica, ecológica y genética. A pesar de estas consideraciones, en los últimos años se planea realizar un megaproyecto denominado Hidroeléctrica El Paso, que abarcaría a los municipios de Pandi, Venecia, Cabrera (en Cundinamarca) e Icononzo (en Tolima). Este potencial proyecto ha generado procesos de organización campesina como forma de resistencia por parte de las comunidades de estos municipios, pues argumentan que un megaproyecto de este tipo no les representa ningún beneficio en zonas con tradición e historia de producción agropecuaria y que, además, se compromete dicha vocación y la riqueza ambiental de la región.

El proyecto hidroeléctrico representa actualmente el conflicto ambiental, socioeconómico y cultural de mayor tensión para la población de Cabrera, debido a la magnitud de sus posibles impactos; sin embargo, este no es el único conflicto ambiental identificado en el proceso de investigación, pues además se evidencian problemáticas comunes en zonas rurales colombianas, como lo son las afecciones a diversas escalas, producto de prácticas agropecuarias convencionales basadas en el uso de tecnologías y agroquímicos. También se presentan relaciones conflictuales heredadas principalmente por las disputas políticas, de las cuales derivan lógicas particulares en la concepción del territorio.

De acuerdo con lo anterior, y a propósito del actual proceso de negociación que vive Colombia entre el Gobierno nacional y la guerrilla de las Fuerzas Armadas Revolucionarias de Colombia (FARC-EP), se han logrado realizar diversas estrategias de acción mancomunada entre organizaciones locales y nacionales, con el apoyo de instituciones educativas. Este es el caso del proceso liderado desde el 2012 por el grupo de investigación Ciudadanía, Paz y Desarrollo de la Corporación Universitaria Minuto de Dios (UNIMINUTO) y el Departamento de Lenguajes y Estudios Socioculturales de la Universidad de los Andes, en conjunto con la comunidad de Cabrera, para llevar a cabo aportes concretos en torno al cumplimiento del Plan de Desarrollo Sostenible planteado por el municipio. Este plan busca el fortalecimiento de la figura de la ZRC y, particularmente, la garantía del equilibrio entre las actividades humanas y el cuidado y mantenimiento de los servicios ecosistémicos.

Polisemia No. 19, 43 - 57. Luchas campesinas y resistencia frente a los conflictos ambientales en la Zona de Reserva [...]. Bogotá, ISSN: 1900-4648. Enero - junio de 2015 
La adaptación de la figura de ZRC para Cabrera resulta trascendental, en la medida en que la población hace uso de esta como su carta de navegación para la organización y consolidación del territorio, en función de seguir el legado de las luchas campesinas propias de esta región, desarrolladas a lo largo del siglo XX en pro de la defensa de su autonomía. Actualmente, las organizaciones locales le apuestan a los medios de concertación posibilitados jurídicamente por la Ley 160, de manera que la expectativa es lograr, por vías consensuadas, la implementación de proyectos locales con proyección regional, a la vez que ser ejemplo de lucha por un territorio que crea su propio ideal de paz.

\section{Contexto de la Zona de Reserva Campesina de Cabrera}

Las ZRC en el país fueron concebidas por la necesidad de dar prioridad a la organización de ciertos territorios marcados por diversos tipos de tensión, principalmente por la presencia del conflicto armado, que tenía - y tiene- como una de sus causas el problema de la repartición inequitativa de la tierra, la cooptación de los territorios y las vulneraciones a los derechos campesinos sobre la tenencia de estos.

La configuración territorial de Colombia se basa en la lógica de la propiedad en su forma capitalista, que hoy se expresa en la forma neoliberal, lo cual, a su vez, se complejiza por las estructuras heredadas del colonialismo. Así, pues, la idea de latifundio - que se traduce en la acumulación de terrenos y el trabajo de la tierra - es infundada o dirigida por una vocación preestablecida desde agentes externos a los productores campesinos locales (pequeños y medianos). En la actualidad, dicha lógica sigue presente y se impulsa de manera sistémica, aun cuando desde finales del siglo $\mathrm{XX}$, con la creación de la actual Constitución de

Figura 1

Área de estudio en el municipio de Cabrera, Cundinamarca

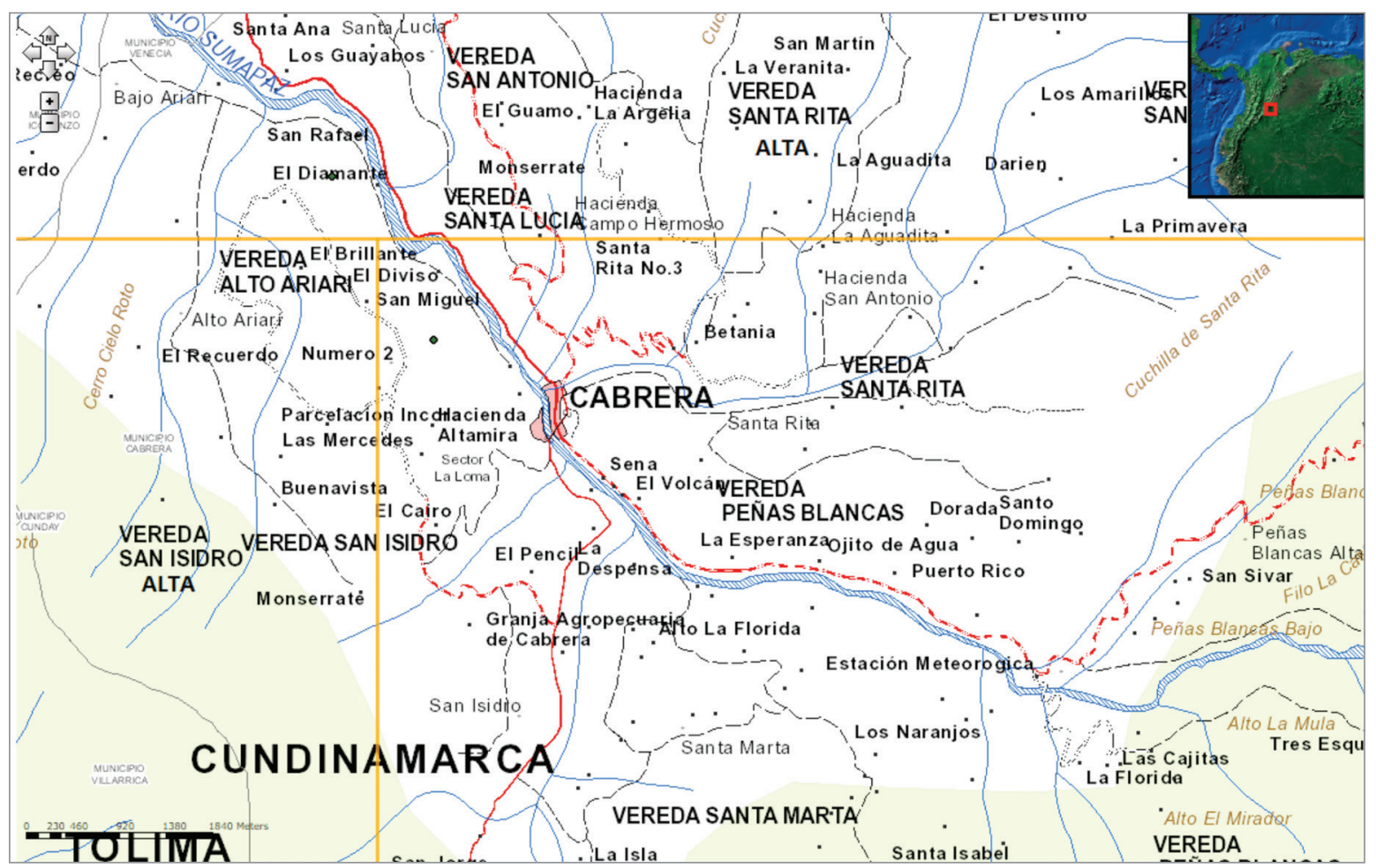

Fuente: Google Maps (2014) 
1991, se hizo un alto en el camino y un reconocimiento a esa vulneración de derechos para comunidades y pueblos (originales) indígenas, campesinos y afrodescendientes (Fajardo, 2006).

El municipio de Cabrera ha sido un lugar precursor en la lucha en pro de los derechos campesinos por la tenencia de la tierra, y así ha generado un legado para el movimiento campesino, arraigado a la idea de proteger, apropiarse y dignificar el territorio habitado. Además de Cabrera, existen otros ejemplos de resistencia y lucha evidenciadas en el ámbito territorial que se han reivindicado por medio de diferentes mecanismos. En este contexto, precisamente la figura de ZRC, como aparece en la Ley 160, se considera un camino propicio para lograr una reforma agraria en el país. Asimismo, al ser este un antecedente del reconocimiento de la problemática generada por el acaparamiento de tierras, también es una medida que busca disminuir (acabar) la situación de violencia infundada básicamente en los principios de tenencia de la tierra por hechos de fuerza.

Cabrera es declarada Zona de Reserva Campesina en el 2000 a partir de la Resolución 046 del 7 de noviembre. Está constituida por 44.000 hectáreas, que abarcan una población aproximada de 5300 habitantes (Instituto Colombiano de Desarrollo Rural [Incoder], 2012, p. 28). Es la única ZRC que está conformada por la totalidad del territorio, incluyendo el área de casco urbano, más las veredas que componen al municipio. La organización que lidera su conformación es el Sindicato de Pequeños Agricultores de Cundinamarca (Sinpeagricun), que manifiesta su interés por mantener la lucha campesina antecedente en el municipio, tendiente a una solución estructural al conflicto social y armado, para poder garantizar una producción agrícola y pecuaria alternativa acorde con las necesidades del campesinado de Cabrera.

La construcción del territorio de manera alternativa frente a la dinámica implantada por el Estado siempre ha sido una característica de la consolidación de la figura de ZRC en el país. Para el caso de Cundinamarca, Cabrera representa, sin duda alguna, un territorio precursor de procesos de resistencia, que se caracteriza por la búsqueda constante de iniciativas que aporten al desarrollo local, y para ello se han tenido como base las necesidades propias y su relación con las demandas del mercado externo que se rigen por la lógica de la globalización.

A sabiendas de que la ZRC es una figura de orden jurídico para el ordenamiento de la propiedad rural, la regulación, la delimitación y la búsqueda de no acaparamiento o acumulación de tierras (Incoder, 2012), y pretendiendo seguir las necesidades y sugerencias de la comunidad del municipio de Cabrera (enmarcadas de igual manera en el Plan de Desarrollo Sostenible), se pretende dar continuación a ese estudio detallado; para este caso específico, identificar las características ambientales de la zona, desde una perspectiva geográfica en el ordenamiento del territorio de Cabrera.

\section{Conflictos ambientales y la Zona de Reserva Campesina de Cabrera}

La imposición directa e indirecta por parte de actores nacionales y externos en cuanto al carácter y la vocación del territorio rural ha "sembrado" en el país, aparte de situaciones de desigualdad e injusticia social, una transformación paulatina en la manera de relacionamiento con el territorio, así como la percepción que se tiene de este y, por ende, el uso que se le da. De esta manera, surgen algunas tensiones o situaciones que resultan conflictuales en las regiones rurales de Colombia, y allí uno de los principales reflejos de los cambios en la relación de las personas con el medio que les rodea se da en el plano ambiental. Ello se determina por la introducción de técnicas y estrategias en el uso del suelo rural diferentes a las tradicionales, así como por la explotación indiscriminada de materias primas, recursos y servicios ecológicos a merced de las solicitudes económicas.

El municipio de Cabrera, además de contar con una importante historia en defensa del te-

Polisemia No. 19, 43 - 57. Luchas campesinas y resistencia frente a los conflictos ambientales en la Zona de Reserva [...]. Bogotá, ISSN: 1900-4648. Enero - junio de 2015 
rritorio campesino, tiene ciertas ventajas únicas relacionadas con sus componentes biológicos y ecosistémicos, determinados por sus características espaciales. Se ubica dentro de la provincia del Sumapaz, que, entre sus generalidades, cuenta con variedad de pisos térmicos, formación de suelos ricos en nutrientes, diversos afluentes hídricos y gran abundancia de especies de flora y fauna. Son características geográficas favorables de Cabrera su ubicación dentro de la cordillera Oriental y sus pisos climáticos que van de templado a frío y a subpáramo, lo que posibilita la presencia de diversas especies de plantas reflejadas de manera clara en la numerosa cantidad de cultivos que han tenido lugar en el municipio.

En este marco, el conflicto ambiental puede ser considerado, en primera instancia, como un conjunto de situaciones de tensión derivadas del ámbito social. Ello corrobora el estado de las relaciones espaciales en torno al ambiente, bien sea por alianzas o diversos niveles de cooperación, o bien, por relaciones surgidas de la diferencia de posiciones o intereses frente al control y manejo de recursos y bienes (Walter, 2009).

También puede referirse el concepto de conflicto ambiental como "aquel que se origina a partir de un impacto, daño o problema ambiental (todas estas expresiones usadas casi como sinónimos) que involucra dos actores cuyos intereses respecto de dichos impactos son contrapuestos" (Padilla, 1990, citado en Folchi, 2001). No se hace necesario que la definición de un conflicto ambiental esté trazada por una situación de afección en la que un actor incida sobre otro; basta con que se reflexione acerca de la presencia de un daño directo o indirecto en los componentes ambientales de un ecosistema para que se considere ello como conflicto ambiental.

La figura de la ZRC busca precisamente consolidar una posición frente al ordenamiento del ambiente, promoviendo la protección y el aprovechamiento óptimo de recursos ambientales; por ello, se hace imprescindible realizar avances propuestos desde la comunidad para una identi- ficación de conflictos ambientales, así como la solución adecuada para estos.

Se considera que la política del lugar y del espacio se basa en las lógicas culturales de las comunidades, en función de convertirse en reclamo social que ayude a legitimar normas democráticas plurales indispensables en la convivencia. Se concibe, entonces, como una necesidad primaria la intervención directa en el territorio, para así atender los distintos ámbitos que forman parte de la construcción de identidad de un pueblo, y entre ellos es crucial el económico, pero viabilizado en torno a la economía local por encima de la global (Leff, 2000, p. 64). De esta manera pueden replantearse nuevas formas de acceder y de trabajar con la comunidad, respetando sus lógicas y retomando los procesos de resistencia campesina que se han adelantado, en este caso, en el municipio de Cabrera, donde se busca como meta común la protección de la autonomía y la defensa y el fortalecimiento de la identidad territorial.

Así se enlazan el acercamiento y trabajo conjunto con la población de Cabrera (liderada por el Comité de Impulso de la ZRC de este municipio) para la generación de estrategias que viabilicen la recolección de información que permita consolidar un diagnóstico conjunto y participativo. En esta línea, el grupo de trabajo constituido entre UNIMINUTO, la Universidad de los Andes, los estudiantes de otras universidades (como la Universidad Distrital Francisco José de Caldas y la Universidad Nacional de Colombia) y el Comité de Impulso se propuso trabajar desde la metodología investigación-acción participativa (IAP) como directriz principal del proyecto, en función de recoger, como fuente del trabajo, los intereses, las necesidades y las prioridades de la comunidad campesina, plasmadas en su actual Plan de Desarrollo Sostenible.

Como parte de este trabajo se consiguió determinar ciertas variables pertinentes para el análisis ambiental, que permitieron identificar los principales conflictos ambientales de la zona con base en las experiencias de la comunidad 
cabreruna. Además, la comunidad muestra implícitamente su percepción sobre el ambiente, sus componentes y afecciones, bien sea a escala micro o macro (en referencia a la totalidad de un ecosistema).

Los conflictos ambientales identificados pueden sintetizarse en: pérdida de la biodiversidad, contaminación de las fuentes hídricas y de los suelos, expansión de la frontera agrícola, introducción de estudios para la realización de megaproyectos y proyectos de extracción, y cambio en las condiciones climáticas.

Es importante resaltar que dentro de esta categorización de conflictos ambientales, la pobla- ción menciona el proyecto hidroeléctrico como el más problemático, debido a que el grado de intervención es externo, mientras que los demás conflictos ambientales tienen una connotación interna (aun cuando también puedan revestir influencia externa).

La figura 2 muestra la cuenca del río Sumapaz que pretende ser intervenida por la multinacional Emgesa, con sus ocho microcentrales. Igualmente se resalta un área definida para la reforestación, lo cual claramente va en contravía de algún proyecto minero-energético. Se podría deducir, además, que de por sí esta es un área afectada por la deforestación, la pérdida de ecosistemas y los deslizamientos de suelo (figura 3).

Figura 2

Cartografía temática: municipios que serán intervenidos

por el proyecto hidroeléctrico de Emgesa, con su respectiva cuenca

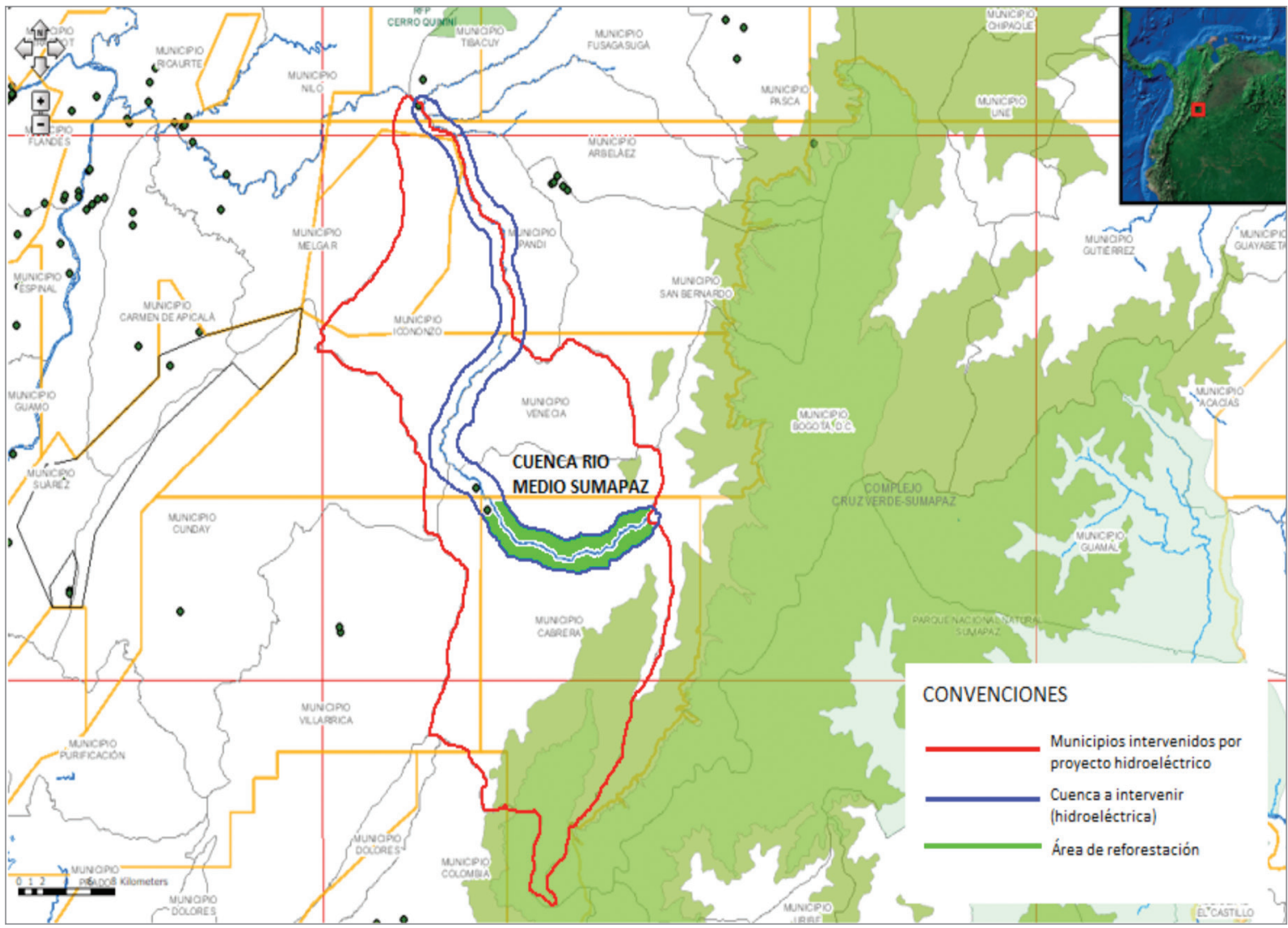

Fuente: elaboración propia a partir de Googles Maps (2014)

Polisemia No. 19, 43 - 57. Luchas campesinas y resistencia frente a los conflictos ambientales en la Zona de Reserva [...]. Bogotá, ISSN: 1900-4648. Enero - junio de 2015 
Figura 3

Cartografía temática: problemas ambientales

y factores de riesgo encontrados en la Zona de Reserva Campesina de Cabrera, Cundinamarca

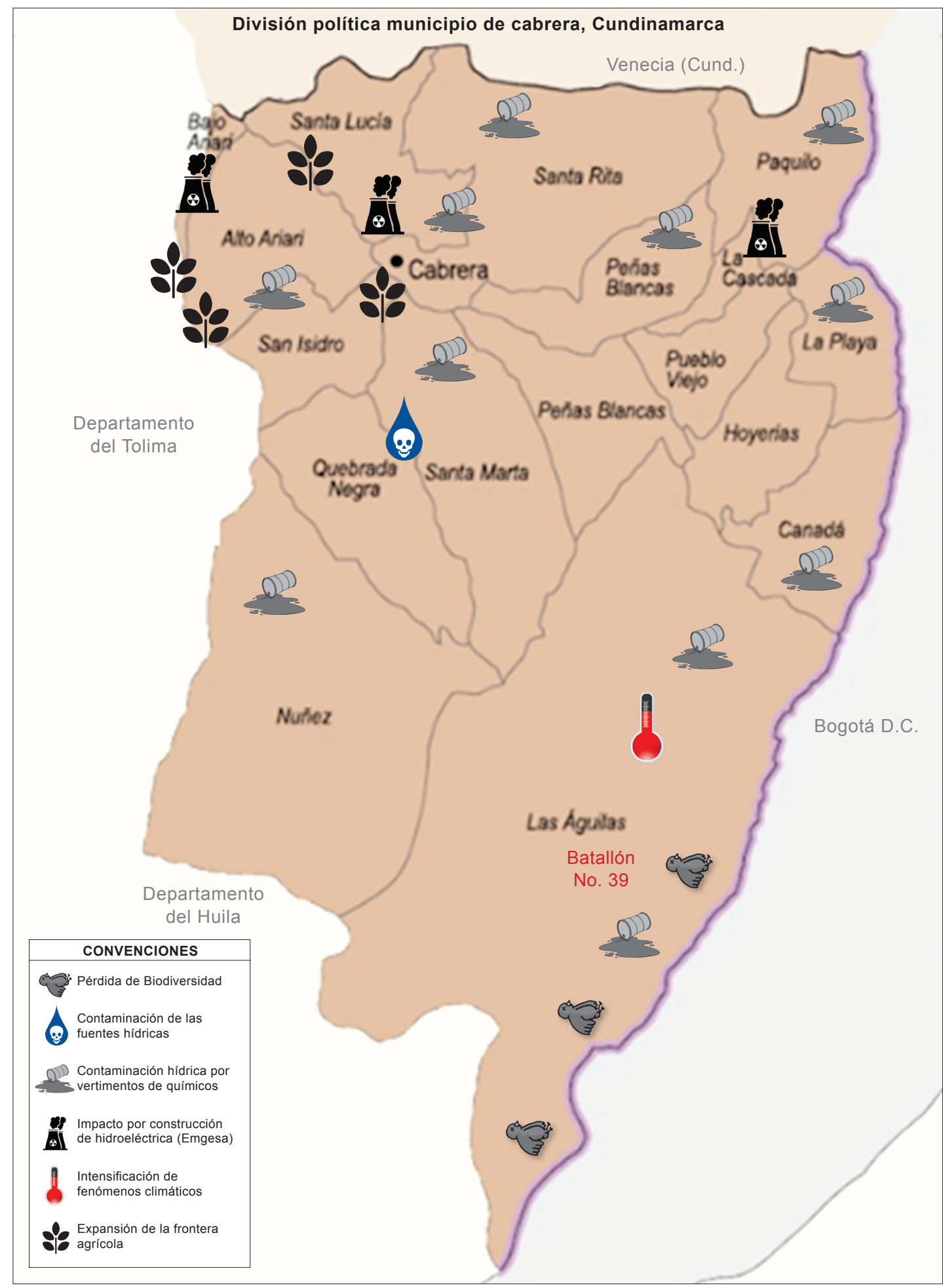

Fuente: elaboración propia (2014). 


\section{Actores y sujetos de los conflictos ambientales}

Los actores identificados en el proceso de investigación como protagonistas de los conflictos ambientales son ciertas instituciones estatales y regionales que, se presupone, son las encargadas del manejo de todas las instancias que cubren la organización y prevención ambiental del municipio. También se identificar algunas instancias internacionales y locales. La categoría actores integra cada uno de los entes, los individuos, las organizaciones y demás figuras que forman parte activa del municipio y que, específicamente, se han visto inmersos en las situaciones que generan conflictos ambientales, bien sea como interventores o afectados (tabla 1).

La figura 4 presenta las organizaciones que forman parte de la ZRC de Cabrera, así como sus alcances o dependencias territoriales. De igual manera, allí se encuentran algunos de los actores identificados en el manejo o la intervención (directa o indirecta) de las temáticas ambientales; para este caso, las asociadas específicamente a los conflictos ambientales presentes en Cabrera.

Tabla 1

Actores protagonistas de los conflictos ambientales en la Zona de Reserva Campesina de Cabrera

\begin{tabular}{|c|c|}
\hline Tipo de actor & Nombre \\
\hline Internacionales & $\begin{array}{l}\text { - Empresa Generadora de Energía S. A. (Emgesa) } \\
\text { - Instituto Latinoamericano para una Sociedad y un Derecho Alternativos (ILSA) }\end{array}$ \\
\hline Nacionales & $\begin{array}{l}\text { - Instituto Colombiano de Desarrollo Rural (Incoder) } \\
\text { - Batallón de Infantería 39, Sumapaz } \\
\text { - Asociación Nacional de Zonas de Reserva Campesina (Anzorc) } \\
\text { - Corporación Universitaria Minuto de Dios (Uniminuto) } \\
\text { - Universidad de los Andes }\end{array}$ \\
\hline Departamentales & $\begin{array}{l}\text { - Gobernación de Cundinamarca } \\
\text { - Corporación Autónoma Regional de Cundinamarca }\end{array}$ \\
\hline Subregionales & $\begin{array}{l}\text { - Sindicato de Pequeños Agricultores de Cundinamarca (Sinpeagricun) } \\
\text { - Comité de Impulso de Zona de Reserva Campesina de Cabrera, Cundinamarca } \\
\text { - Unidad Municipal de Asistencia Técnica Agropecuaria (Umata) } \\
\text { - Comité de Jóvenes en Defensa del Río Sumapaz } \\
\text { - Asociación de Juntas de Acción Comunal } \\
\text { - Gremios de productores y productoras }\end{array}$ \\
\hline
\end{tabular}

Fuente: elaboración propia (2014). 
Figura 4

Nodos de actores y entes vinculantes

en la Zona de Reserva Campesina de Cabrera, Cundinamarca

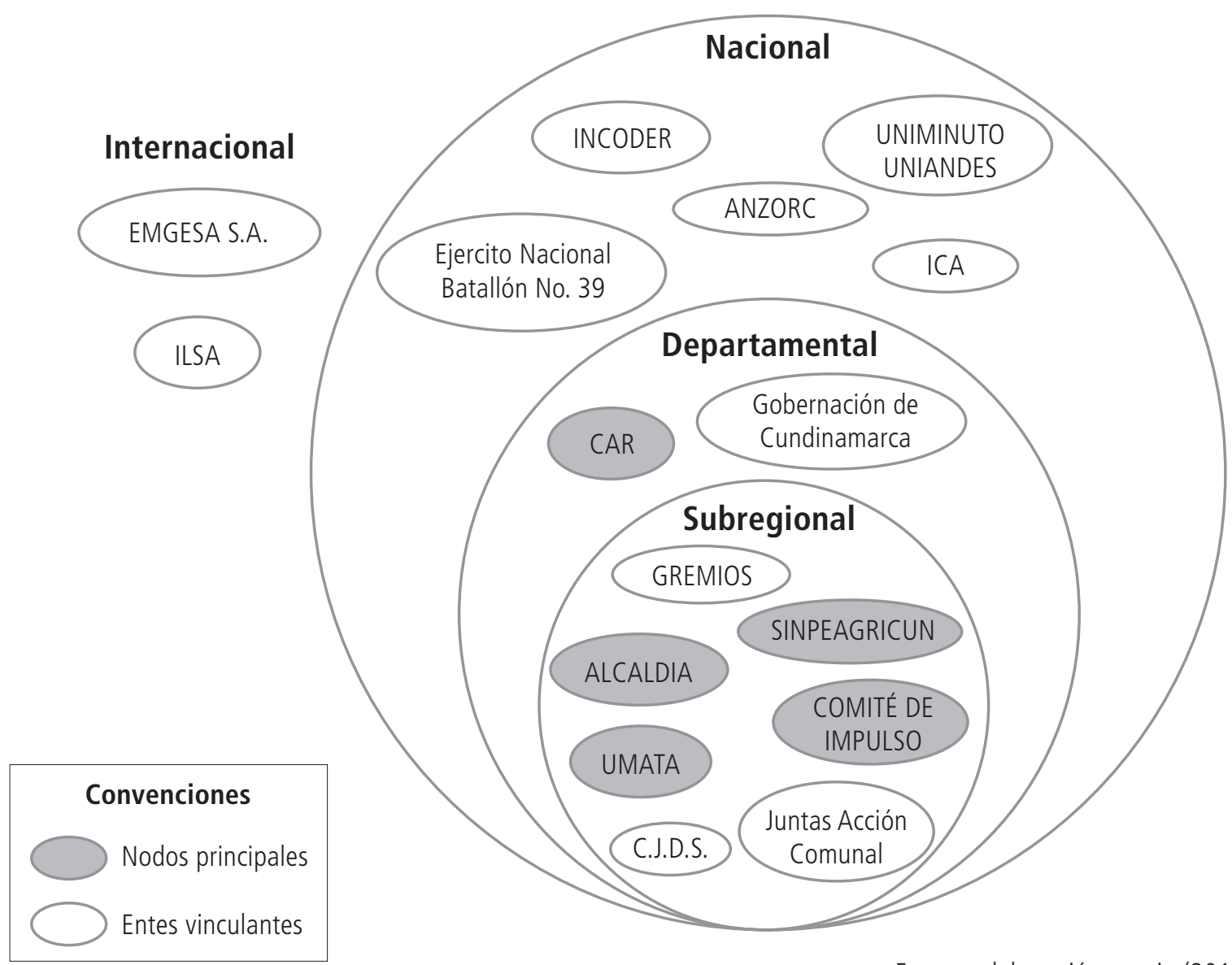

Fuente: elaboración propia (2014).

\section{Prácticas y estrategias de resistencia}

En el proceso de investigación también se identificaron una serie de prácticas y estrategias que se pueden catalogar como avances e iniciativas realizados por los diversos actores inmersos en los conflictos ambientales de la ZRC de Cabrera. Estas prácticas tienen como objetivo fundamental hacer resistencia y buscar alternativas frente a las afecciones presentes en el territorio derivadas de dichos conflictos. Pueden caracterizarse de la siguiente manera las prácti- cas que han tenido mayor impacto en el territorio, en cuanto a su acogida o persistencia en el territorio:

a) Acciones de movilización. Se propone esta subcategoría para poder clasificar los hechos que representen acciones de visibilización de los conflictos ambientales de Cabrera, así como gestiones de denuncia pública frente a situaciones puntuales que, se considera, deben ser conocidas por el grueso de la población y ser atendidas por las entidades e instituciones nacionales pertinentes. Dentro de las acciones de movilización se sitúan las asambleas, 
los encuentros, los foros, las alianzas con agentes externos, las socializaciones, las marchas y los plantones que han realizado la comunidad para dar a conocer sus preocupaciones. Para este caso, principalmente se encuentran las acciones enmarcadas dentro de la atención especial al conflicto ambiental que representa la creación de la Hidroeléctrica El Paso por parte de Emgesa.

b) Acciones de activación eintervención. Aquí se describen las acciones puntuales que han buscado contrarrestar directamente alguno de los conflictos ambientales presenciados y que han pretendido o logrado la transformación de algún conflicto ambiental presente en la ZRC de Cabrera. Puede catalogarse aquí el uso de recursos legales, las denuncias, los talleres, las jornadas de sensibilización y la elaboración de documentos.

Como fruto de las estrategias de resistencia de las organizaciones, el Plan de Desarrollo Sostenible de la Zona de Reserva Campesina de Cabrera, Cundinamarca (2013) se considera el documento más importante hasta ahora creado por la comunidad campesina. Este fue liderado por Sinpeagricun, con apoyo estatal del Incoder y mediante alianzas externas con el Instituto Latinoamericano para una Sociedad y un Derecho Alternativos (ILSA). Este documento se plantea como la compilación de años de trabajo, de luchas campesinas, de su historia y su contexto, y también como el diagnóstico de las principales características (componentes: de ordenamiento ambiental territorial, social, productivo y de tierras) y de las proyecciones futuras en lo que ha implicado - e implica aún - la construcción territorial de Cabrera.

Otro de los documentos generados en la comunidad de Cabrera es la Declaratoria Sumapaz en debate: campesinos y ambientalistas en defensa del territorio y del agua (2014), realizada y facilitada por el Sindicato de Trabajadores Agrícolas del Sumapaz (Sintrapaz), a modo de denuncia pública de las actuales preocupaciones frente al in- minente daño que causarán las microcentrales hidroeléctricas en áreas de la provincia el Sumapaz. Igualmente se hace un llamado a la reflexión y a la participación de toda la sociedad colombiana en una lucha por los derechos, los recursos y la autonomía territorial que se está perdiendo, al darle avales y terrenos que no les corresponden a empresas extranjeras como Emgesa.

Resultado de esas múltiples tareas o prospectivas consignadas en el PDS de la ZRC de Cabrera, se realizan todo tipo de alianzas avaladas por las respectivas organizaciones locales. $\mathrm{La}$ participación de instituciones académicas es una de las estrategias más convincentes por personas de la comunidad. En específico, a propósito de este trabajo investigativo se desarrolló el "Taller de agroecología con la comunidad de Cabrera, Cundinamarca", liderado por el programa de Ingeniería Agroecológica de UNIMINUTO. El objetivo del taller fue "construir de manera participativa un referente desde el cual se puedan planear y desarrollar los sistemas de producción agropecuarios en la Zona de Reserva Campesina, que contribuyan al cumplimiento del Plan de Desarrollo Sostenible". Los alcances de dicho taller han significado, hasta el momento, un diagnóstico constituido por un recuento histórico del territorio y la identificación de problemáticas y alternativas dentro de los componentes ambientales, económico-productivos, socioculturales y político-territoriales.

Asimismo, el Comité de Impulso, con apoyo de UNIMINUTO y de Uniandes, produjo a finales de 2014 el video documental En defensa del rio Sumapaz, instrumento de visibilización de la posición de la comunidad campesina de Cabrera frente al proyecto hidroeléctrico antes mencionado, además de buscar fortalecer la perspectiva de trabajo conjunto entre estas instituciones y organizaciones ${ }^{3}$. Este video ha tenido gran acogida entre diversos organismos y escenarios nacionales e internacionales.

3 Los resultados del trabajo realizado en el 2014 fueron socializados con la comunidad el 20 y 21 de febrero de 2015. Asimismo, el 10 de julio del 2015 se realizó un cine-foro en la plaza de mercado del casco urbano de Cabrera.

Polisemia No. 19, 43 - 57. Luchas campesinas y resistencia frente a los conflictos ambientales en la Zona de Reserva [...]. Bogotá, ISSN: 1900-4648. Enero - junio de 2015 
Otra de las estrategias que se han llevado a cabo desde el 2012, y acorde con el Plan de Desarrollo Municipal, es el programa liderado por la Umata: Descontaminación del Medio Ambiente, el cual cuenta con intereses mancomunados con la comunidad para realizar procesos de reforestación, conservación y adquisición de predios de interés ambiental, así como estrategias en cuanto al uso, la ocupación y el manejo del suelo.

La dificultad más grande para que se ejecuten este tipo de proyectos o iniciativas es la falta de asesoría para su consolidación adecuada, pues a pesar de que según el Plan de Desarrollo Sostenible los recursos existen o pueden ejecutarse, si esto no se hace de la manera adecuada, lo más probable es que la financiación sea desaprovechada.

\section{La Zona de Reserva Campesina de Cabrera, alternativa territorial para la paz}

Desde sus inicios, el municipio de Cabrera ha sido precursor en el uso de estrategias pertinentes en defensa de los derechos de su comunidad. De hecho, ha extendido esas intencionalidades con miras al aporte regional y, por supuesto, nacional. La lucha campesina, que aboga por los derechos plurales que se enmarcan dentro de la lógica territorial propia, hace de Cabrera un municipio visionario frente a lo que significa la utilización y construcción de esas herramientas jurídicas, para generar procesos tanto de reconocimiento en el ámbito nacional como de fortalecimiento en el local.

La atención a las necesidades propias del municipio, por medio de instrumentos de construcción colectiva como el PDSS-ZRC, da cuenta de la organización existente en Cabrera y de la importancia que ello representa. De igual manera, las organizaciones locales son conscientes del valor de generar alianzas con entes externos que puedan orientar las acciones por realizar en ma- teria ambiental, para que ello sea acorde con los propósitos y las metas que tiene la comunidad de Cabrera. Se parte de los intereses desde la figura de ZRC, así como desde el ordenamiento de su componente ambiental y teniendo en cuenta el conflicto más latente actualmente, que es la proyección de la Hidroeléctrica El Paso. Así se demuestra una vez más que un seguimiento juicioso y detallado, por diferentes medios de resistencia abanderados por la figura de ZRC constituyen mecanismos en pro de la paz, y ello forma parte del legado de luchas regionales que alguna vez radicaron en el uso de la fuerza y el posicionamiento político, hasta llegar a la búsqueda incansable de acuerdos y mediaciones por medio del diálogo y la consulta. Aunque resulta difícil poder debatir siquiera con multinacionales como Emgesa, los habitantes de Cabrera demuestran que hacerse escuchar resulta trascendental si existe constancia y unidad, y sobre todo cuando se realiza una gestión continua con diferentes entes aliados y con más de una estrategia a bordo.

Al igual que las diferentes características (materiales o no) que componen los territorios, las intervenciones de solución deben ser igualmente tratadas, de ser posible con cada uno de los habitantes, reflexionando sobre la incidencia que su actuar tiene o puede tener sobre el medio. La ZRC es entonces una buena muestra de lo que puede llegar a significar la paz en un territorio, pues las iniciativas mancomunadas y los esfuerzos colectivos pueden recrear espacios óptimos para la participación, para servir a la atención de necesidades comunitarias propias bajo una mirada integral y colectiva.

El buen uso de lo que consolidaría una figura jurídica como la ZRC puede ser la única opción que encuentran ciertas comunidades que han sido víctimas de la situación de violencia y desigualdad en Colombia. Su territorio llega a representar casi el único modo de subsistencia; pero este ha sido permeado por "nuevas" lógicas económicas que deterioran sustancialmente lo existente a su paso, sobre todo la dimensión física, sin dejar de lado todo el tejido social, las costumbres y la tradiciones de una comunidad que 
desea perdurar en el tiempo y convertirse o, más bien, seguir siendo ejemplo de lucha e identidad.

El ejemplo de la actual resistencia de Cabrera frente al megaproyecto hidroeléctrico resulta ser un reflejo a escala de la situación actual del país, de la situación del campesinado colombiano. Y aunque se ha hecho caso omiso a las solicitudes de la población cabreruna, aun cuando se supone se tiene una fuerte posibilidad de "ganancia" jurídica por ser una ZRC, ¿qué puede esperarse entonces para el resto de poblaciones que sufren situaciones similares, pero donde no existe figura tal que las cobije?

$\mathrm{Al}$ ser Cabrera el único municipio del departamento de Cundinamarca en formar parte de la figura de la ZRC, es también punto de referencia trascendental en lógicas de desarrollo territoriales diferentes a las tradicionales, pues es este sin duda uno de los ejes regionales del centro del país, por sus importantes avances y nuevas perspectivas. Ello podría presuponer una reserva pionera que aporte en la construcción de la idea de crear la ZRC del Sumapaz, localidad 20 de Bogotá, como se ha venido proponiendo para la capital del país, al igual que la planeación de la ZRC para el municipio (también vecino) de San Bernardo, Cundinamarca.

Lo anterior da cuenta de los importantes cambios en las nociones que se están construyendo sobre el territorio, así como las estrategias ideadas para apropiarse de ente. En este contexto debe entenderse el papel que desempeña el individuo como ser perteneciente a una comunidad; todo esto a raíz de la implementación y adopción de la figura de ZRC.

\section{Conclusiones}

Históricamente, la configuración territorial de Colombia se ha caracterizado por vivenciar diversos conflictos agrarios cuya solución planteada son mecanismos de orden jurídico que buscan ordenar y normatizar el uso del terreno en pro de intereses comunes. Estos mecanismos, lamentablemente, en la práctica no corresponden a la realidad del país, sino que más bien responden a lógicas y solicitudes de carácter externo a escala mundial.

Una de las grandes preocupaciones de la actual organización del territorio nacional es el componente ambiental. Es este uno de los puntos claves que requieren atención precisa de todos los entes territoriales competentes, sin que ello excluya a las organizaciones locales y a las comunidades otras involucradas en la construcción, la planeación, la ejecución y el monitoreo de procesos que contribuya a solucionar cada una de las problemáticas identificadas en los diferentes contextos. La retroalimentación y las alianzas son entonces piezas claves dentro de esas nuevas alternativas territoriales que han encontrado las comunidades.

Al igual que las organizaciones locales, los gremios y las juntas de acción comunal de Cabrera han velado por mantener una interacción constante y activa con la comunidad, al hacerles partícipes de la mayoría de procesos e iniciativas. Así, el trabajo que realicen los actores externos debe ser conjunto con los actores internos o locales, pues aunque pareciese obvio, no siempre se han propiciado adecuadamente los espacios ni se han brindado las herramientas necesarias para la participación activa de todos los actores inmersos en los diferentes conflictos. Este es el caso claro de la multinacional Emgesa, que ha sido fuertemente criticada por hacer partícipe a la comunidad únicamente con pequeñas socializaciones, aunque con información indefinida y omitiendo completamente la versión, el testimonio y las pruebas de quienes están en desacuerdo con llevar a cabo dicho megaproyecto hidroeléctrico en una zona de vocación agrícola y pecuaria.

De igual forma, se crea el Plan de Desarrollo Sostenible de la Zona de Reserva Campesina de Cabrera, Cundinamarca, documento que contiene cada elemento relevante para la comunidad interesada en formar parte del ordenamiento

Polisemia No. 19, 43 - 57. Luchas campesinas y resistencia frente a los conflictos ambientales en la Zona de Reserva [...]. Bogotá, ISSN: 1900-4648. Enero - junio de 2015 
de su territorio, donde de manera autónoma y consensuada se encaminan las actividades económicas, culturales, sociales y ambientales del municipio.

Las ZRC desempeñan un papel fundamental en la gestión autónoma del territorio, pues representa una figura útil al momento de plantear alternativas viables para la gestión de desarrollo municipal. Estas son empleadas como "carta de navegación" frente a los procesos y las características ambientales, así como respecto a los posibles manejos que se le pueden dar a las situaciones conflictuales de dicho ámbito. Ante la inoperancia de las soluciones propuestas por los diferentes gobiernos, el movimiento campesino, ubicado especialmente en zonas de conflicto territoriales (como en el caso del municipio de Cabrera), ha logrado articular la construcción de mecanismos propios de autogestión, defensa del territorio, consecución de recursos y atención a las necesidades de carácter social. El PDS de la ZRC de Cabrera deviene en un documento fundamental, además por ser la plataforma en la que se plantea la posibilidad de trabajar en nuevas alternativas con agentes externos interesados, como, en este caso especial, UNIMINUTO y UniAndes.

La ZRC de Cabrera es una muestra de la consolidación organizativa dada por sus antecedentes históricos de luchas campesinas agrarias en la región. Su implementación ha logrado consolidar procesos comunitarios previos y visibilizar a escalas más grandes las dificultades y los retos que existen en el municipio y que conciernen a toda una región, como lo es la provincia del Sumapaz.

El conjunto de acciones que componen o han formado parte de la construcción de la figura de ZRC tanto en Cabrera como en los demás municipios de Colombia es un reflejo de los movimientos de resistencia y de las acciones propias de las organizaciones locales de diversa índole generadas por las poblaciones del país. La constante búsqueda de reconocimiento de derechos fundamentales, así como la necesidad de crear rutas autónomas propias que integren las tradiciones con la realidad actual de los territorios, es un indicador del viraje que ha producido la constante imposición nacional por determinar las capacidades y posibilidades de los territorios, situación que ha dejado desarraigo, desplazamiento, violencia, pobreza y desigualdad para el campesinado colombiano.

A pesar de que las situaciones lamentables han sido numerosas para las comunidades, a tal punto de llegar a ser difícilmente reparables, las poblaciones han decidido apersonarse de los procesos transformadores de sus realidades; han entendido que la salida es el ordenamiento autónomo del territorio para lograr determinar adecuadamente las acciones que resulten ser funcionales y que integren las distintas esferas de una sociedad y comunidad.

La necesidad de superar las barreras que la economía tradicional ha puesto a lo largo del territorio rural colombiano ha derivado en una exploración de soluciones para los daños palpables, de manera inicial por parte del gobierno; pero en vista de que no resultan ser viables a escala local, las comunidades han decidido hacer uso de ciertas herramientas jurídicas antes mucho más desconocidas y subestimadas, pero que ahora resultan ser ese eje que permite llevar a cabo la autogestión y que además pretende "erradicar" esas acciones que imposibilitan esa búsqueda de paz, armonía, equilibrio y proyección territorial tan primordial en la época actual. Sin adentrar mucho aún en discusión, pareciese que esta iniciativa de creación de reforma agraria determinada por la figura de Zona de Reserva Campesina proyectó de alguna manera ser parte del actual Proceso de Paz que vive Colombia. Es esta esta una evidente iniciativa de ordenamiento territorial integrador, autónomo y adecuado para las comunidades, donde las organizaciones rurales locales y sus procesos de resistencia tienen gran parte del protagonismo y de la responsabilidad en cuanto a la identificación y creación de insumos necesarios en la práctica de esas nuevas alternativas que el campo colombiano desea. 


\section{Referencias}

Alcaldía de Cabrera (s. f.). Unidad Municipal de Asistencia Técnica Agropecuaria. Recuperado de http://www.cabrera-cundinamarca.gov.co/Nues tros_planes.shtml?apc $=\mathrm{I}-\mathrm{xx}--3128021 \& \mathrm{x}=$ 1914146

Colectivo de Abogados José Alvear Restrepo (2014). Páramo de Sumapaz, en la mira de las hidroeléctricas. Recuperado de http://www.colectivodeabogados.org/noticias/noticias-nacionales/ Paramo-de-Sumapaz-en-la-mira-de

Congreso de la República de Colombia (1994). Ley 160 de 1994, por medio de la cual se crea el Sistema Nacional de Reforma Agraria y Desarrollo Rural Campesino, se establece un subsidio para la adquisición de tierras, se reforma el Instituto Colombiano de Reforma Agraria y se dictan otras disposiciones. Bogotá: Autor.

Corporación Autónoma Regional de Cundinamarca (2009) Resnatur, Sumapaz. Bogotá: Autor.

Ejército Nacional de Colombia (s. f.). Batallón de Infantería 39 de Sumapaz, Quinta División. Recueprado de http://www.quintadivision.mil. co/ idcategoria $=277535$

Fajardo, D. (1998). Sistemas de tenencia de tierras de comunidades en el ordenamiento sostenible de los recursos naturales. En Agro y medio ambiente. Bogotá: Fundación Friederich Ebert de Colombia.

Fajardo, D. (2000). Las Zonas de Reserva Campesina: ¿estrategia de desarrollo regional y contra el desplazamiento? Recuperado de http:// www.mamacoca.org/Compendio_regional/ Dario_Fajardo\%20.htm
Fajardo, D. (2006). Zonas de Reserva Campesina, otra experiencia en la briega por la tierra. Recuperao de http://www.scribd.com/ doc/17319863/Las-Reservas-Campesinas

Folchi, M. (2001). Conflictos de contenido ambiental y de ecologismo de los pobres: no siempre pobres, no siempre ecologistas. Recuperado de http://www.fuhem.org/media/ecosocial/File/ Boletin\%20ECOS/Boletin\%206/Conflictos\%20 ambientales_M.WALTER_mar09_final.pdf

Instituto Colombiano de Desarrollo Rural (Incoder) et al. (2012). Zonas de reserva campesina. Elementos introductorios y de debate. Bogotá: Autores.

Instituto Latinoamericano para una Sociedad y un Derecho Alternativos (ILSA) (s. f.). Quiénes somos. Recuperado de http://ilsa.org.co:81/ node $/ 2$

Leff, E. (2000). Espacio, lugar y tiempo: la reapropiación social de la naturaleza y la construcción local de la racionalidad ambiental. Desenvolvimento e Meio Ambiente, n.1. Editora da UFPR

Municipio de Cabrera (2013). Plan de Desarrollo Sontenible de la Zona de Reserva Campesina de Cabrera, Cundinamarca (2013). Cabrera, Colombia: Autor.

Organización de las Naciones Unidas para la Alimentación y la Agricultura (FAO) (2009). Visión prospectiva de la Zona de Reserva Campesina de Cabrera. Bogotá: Autor.

Pérez Martínez, M. E. et al. (2014). Gestión ambiental territorial: dinámicas y trayectorias de la participación ciudadana y sostenibilidad de los recursos naturales en la jurisdicción de la CAR,

Polisemia No. 19, 43 - 57. Luchas campesinas y resistencia frente a los conflictos ambientales en la Zona de Reserva [...]. Bogotá, ISSN: 1900-4648. Enero - junio de 2015 
Cundinamarca: la voz de los actores locales. Bogotá: Pontificia Universidad Javeriana y Corporación Autónoma Regional (CAR).

Sindicato de Pequeños Agricultores de Cundinamarca (Sinpeagricun). Estatutos de Organización. Recuperado de http://sinpeagricun.es.tl/ ESTATUTOS-DE-ORGANIZACI\%D3N.htm

Sindicato de Pequeños Agricultores de Cundinamarca (Sinpeagricun) et al. (2013). Plan de Desarrollo Sostenible Zona de Reserva Campesina de Cabrera (Cundinamarca). Por una zona de reserva campesina garante de derechos económicos, sociales, culturales y ambientales para los cabrerunos (2013). Bogotá: Autores.

Walter, M. (2009). Conflictos ambientales, socioambientales, ecológico distributivos, de contenido ambiental... Reflexionando sobre enfoques y definiciones. Madrid: Centro de Investigaciones para la Paz. 
